\title{
Cross-Correlations of Financial Crises Analysis by Power Law Classification Scheme
}

\author{
J. MiŚKIEWICZ* \\ Institute of Theoretical Physics, University of Wrocław, pl. M. Borna 9, 50-204 Wrocław \\ Department of Physics and Biophysics, Wrocław University of Environmental and Life Sciences, \\ Norwida 25, 50-375 Wrocław

\begin{abstract}
Cross-correlations among the chosen six main world financial markets are analysed by power law classification scheme (PLCS). The markets are represented by indices: DAX (Frankfurt), FTSE (London), S\&P 500 (New York), HSI (Honkong), Nikkei 225 (Tokyo), STI (Singapore) in the interval from 24.09.1991 till 31.01.2014. The time series are transformed into daily returns and normalised daily range of indices. The evolution of correlation strength is analysed using moving time window. It is shown that the correlation strength properly characterises crisis and prosperity periods. Moreover, the value of the correlation strength can be related to the crisis severity. The results are compared with standard ultrametric distance based on Pearson coefficient.
\end{abstract}

DOI: 10.12693/APhysPolA.127.A-103

PACS: 89.65.Gh, 05.45.Tp

\section{Introduction}

The first financial crises was observed in 17-th century as a result of tulipomania [1] and since then the crisis becomes permanent, although unwanted element of stock markets. In fact, financial crashes are not only problem of a narrow group of investors but they can strongly influence a real economy even on a global level. In difference to the 17-th century Europe on the contemporary markets thousands of bonds, shares and other types of securities are traded. Therefore, now the crises is understood as the situation when at the same time significant number of securities lose its values. In such a case essential for the crisis occurrence are the cross-correlations among listed securities. Due to this fact, the recently developed method of nonlinear cross-correlations analysis: power law classification scheme (PLCS) [2,3] is applied to the analysis of correlations between the stock market indices of the main world markets: DAX (Frankfurt), FTSE (London), S\&P 500 (New York), HSI (Honkong), Nikkei 225 (Tokyo), STI (Singapore). The investigated time series cover the period from 24.09.1991 till 31.01.2014, which consists of 5500 data points. The considered indices differ in methodology of calculation therefore, instead of analysing correlations among the values the time series are transformed into the daily returns and normalised daily range of indices and then analysed. Both transformations remove trends, so the main difference between them is the time horizon of measured variability. The daily return measures the changes between consecutive trading days while the normalised daily range inform us about index value fluctuation within the trading day. Therefore, the latter is more sensitive to the

*e-mail: janusz.miskiewicz@ift.uni.wroc.pl rapid fluctuations, which are characteristic for crashes. Since a crisis is not an event but rather a process, so the evolution of correlation strength is analysed. The correlation strength is calculated in moving time window for two time windows lengths: 50 days and 100 days. The results are compared with the standard ultrametric cross-correlations distance measures introduced by Mantegna [4].

\section{Power law classification scheme}

One of the most frequently used measure of crosscorrelations in the field of econophysics is the ultrametric measure developed and successively used by Mantegna and collaborators in series of works e.g. [4-8]. It is based on the Pearson linear correlation coefficient and therefore is focused on linearity of analysed correlations, which in general does not have to be linear. The power law classification scheme was developed as an alternative to the measure proposed by Mantegna. The detailed description of the algorithm as well as its application to the analysis of macroeconomy problems can be found in $[2,3]$. In this paper the next step in establishing the potential area of PLCS application is made. The algorithm is applied to the analysis of financial crises. For the convenience of the reader the algorithm will be shortly described below.

Let denote the analysed time series as $A$ and $B$ and theirs elements as $a_{i}$ and $b_{i}$. It is assumed that $A$ and $B$ were registered at the same moments of time and they have the same number of points (if not - the analysis is performed on the overlapping parts of $A$ and $B$ ). The PLCS algorithm consists of the following 4 steps:

1. Firstly: The cumulative Manhattan distance (MD) between $A$ and $B$ is calculated

$M D(A, B)(j)=\sum_{i=1}^{j}\left|a_{i}-b_{i}\right|, j \in(1,2, \ldots, T)$, 
where $T$ is the number of data points in the analysed time series. Notice that at the series of cumulative MD (Eq. (1)) each sum have the same initial point while the number of the summed elements gives the position of an element in cumulative MD series.

2. At the next step: Present the calculated cumulative MD time series as a function of the element index in $\log -\log$ scale.

3. Finally: Fit the linear function to the data and find the slope coefficient $\gamma$. The correlation strength is defined as $\alpha=\gamma-1$.

4. Additionally, the quality of the fit can be estimated. It is defined by the statistical significance probability $\beta$ of the model. In fact, it provides information about the stability of the correlation in the considered time interval.

The results of correlation strength can be classified into five cases: (i) $\alpha<0$ the time series are convergent, (ii) in the case of $\alpha=0$ they are parallel (linearly correlated), (iii) for $0<\alpha<1$ - slowly divergent, (iv) if $\alpha=1$ divergent but linearly correlated and finally (v) for $\alpha>1$ time series are strongly divergent.

Although the ultrametric distance (UD) is broadly known but for the sake of clarity the key point of its definition will be recalled below. The UD is based on the Pearson linear coefficient, which is defined as:

$$
\begin{aligned}
& P_{(t, T)}(A, B)= \\
& \frac{\langle A B\rangle_{(t, T)}-\langle A\rangle_{(t, T)}\langle B\rangle_{(t, T)}}{\sqrt{\left(\left\langle A^{2}\right\rangle_{(t, T)}-\langle A\rangle_{(t, T)}^{2}\right)\left(\left\langle B^{2}\right\rangle_{(t, T)}-\langle B\rangle_{(t, T)}^{2}\right)}},
\end{aligned}
$$

where $\langle\cdot\rangle_{(t, T)}$ denotes the mean value in the interval $(t, t+T)$. There are two formulations of UD in literature: the introduced originally by Mantegna [4]

$$
\text { or } \begin{aligned}
D U_{M S}(A, B)_{(t, T)} & =\sqrt{2\left(1-P_{(t, T)}(A, B)\right)}, \\
D U_{A M}(A, B)_{(t, T)} & =\sqrt{\frac{1}{2}\left(1-P_{(t, T)}(A, B)\right)},
\end{aligned}
$$

introduced in [9]. Both definitions Eq. (3) and Eq. (4) are in fact equivalent, they differ only in normalizations. While the original formulation Eq. (3) maps the distance between time series into the interval $(0,2)$ the alternative definition Eq. (4) takes the value in the interval $(0,1)$. Within this paper the Eq. (4) will be used and denoted as UD.

\section{Data}

Within this study the analysis of cross-correlations between main world stock markets is performed. The following three global regions are investigated: North America - New York Stock Exchange, Europe — Frankfurt and London stock exchanges, far east — Tokyo, Honkong and Singapore stock exchanges. The state of stock exchanges are described by the indices: DAX (Frankfurt), FTSE (London), S\&P 500 (New York), HSI (Honkong), Nikkei 225 (Tokyo), STI (Singapore). The date were obtained from the Yahoo Finance service. The time series cover the interval from 24.09.1991 till 31.01.2014 i.e. 5500 data points. The plots of the close value of considered indices are presented in Fig. 1.

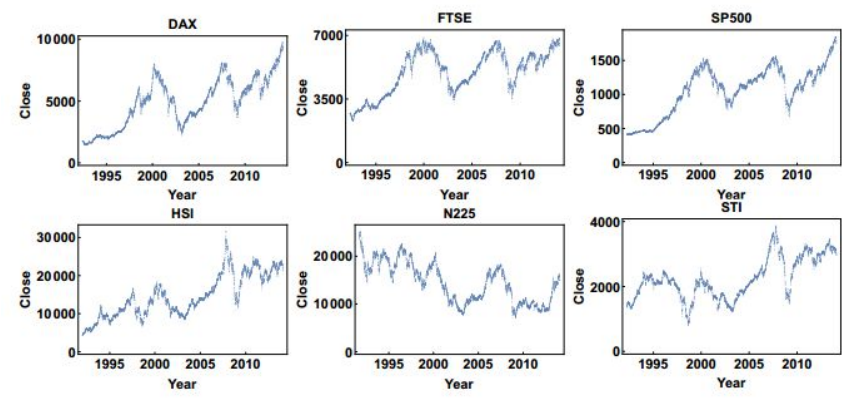

Fig. 1. The evolution of DAX, FTSE, S\&P 500, HSI, Nikkei 225, STI indices in the period 24.09.1991 till 31.01 .2014 .

The time series indices are calculated by different methodology and they differ in values, however it can be suspected that theirs changes should reflect behaviour of the system. Therefore, the analysed time series are transformed into daily returns and normalised daily range of indices. The daily return of an index is defined on the closing values of the index in the standard way:

$$
r a_{t}=\frac{\operatorname{close}\left(a_{t}\right)-\operatorname{close}\left(a_{t-1}\right)}{\operatorname{close}\left(a_{t-1}\right)} .
$$

The normalised daily range is defined as follows:

$$
\Delta_{t}=\frac{\max \left(a_{t}\right)-\min \left(a_{t}\right)}{\operatorname{close}\left(a_{t}\right)},
$$

where close $\left(a_{t}\right)$ is the closing value of the index, $\max \left(a_{t}\right)$ and $\min \left(a_{t}\right)$ are the maximum and minimum of the stock market index during the trading day $t$. The daily return Eq. (5) provides information about index value fluctuation in a daily time horizon while the normalised daily range Eq. (6) is focused on the intra-daily changes. The time series of indices transformed by the daily return and the normalized daily range are presented in Figs.2, 3.
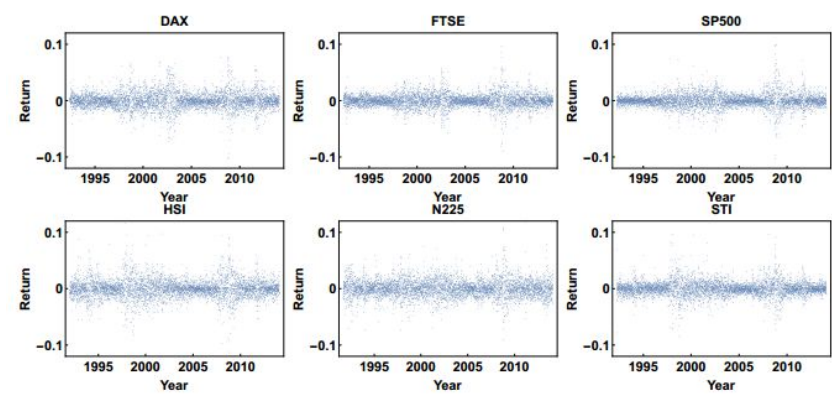

Fig. 2. The evolution of DAX, FTSE, S\&P 500, HSI, Nikkei 225, STI indices transformed into daily return in the period from 24.09.1991 till 31.01.2014. 

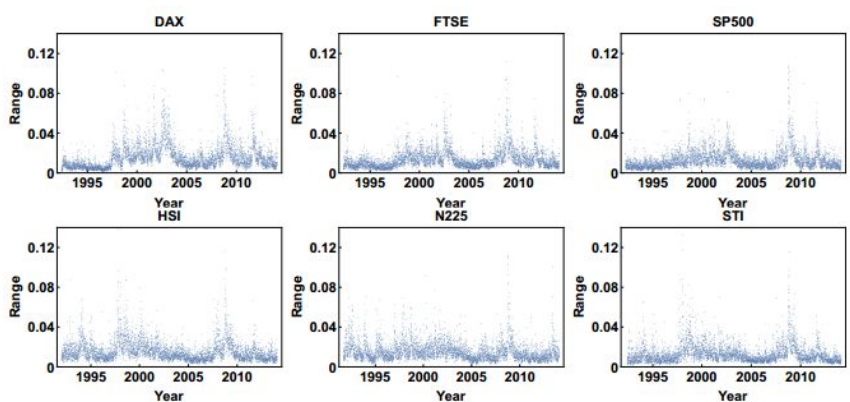

Fig. 3. The evolution of DAX, FTSE, S\&P 500, HSI, Nikkei 225, STI indices transformed into normalised daily range in the period from 24.09.1991 till 31.01.2014.

\section{Results}

The set of analysed time series consists of six indices so the correlation matrix contains 15 unique parameters. Moreover, to obtain the evolution of correlations among the time series the correlation matrices are calculated in a moving time window of size $T$. At each calculation step the time window was shifted by one data point. Two time window sizes were chosen: $T=50$ days (d) and $T=100 \mathrm{~d}$. Since the main aim of the analysis is to verify possibility of PLCS application in distinguishing and recognition of crisis periods on a global level each of the correlation matrix is represented by mean value and standard deviation of unique correlation matrices elements. The correlation stability of the fitted models measured as the statistical significance in all cases did not exceeded $\beta \leq 3.2 \cdot 10^{-15}$, so they can be considered as reliable fits.

The mean value and standard deviation evolution of correlation strength among daily returns and normalised daily range are presented in Figs. 4-7. The red and green arrows indicate examples of well distinguished crises and prosperity periods respectively. Comparing the evolution of the stock markets indices presented at Fig. 1 and the PLCS correlation strength of the returns and normalised daily range time series at Figs. $4-7$ the following observation can be made. At the prosperity periods the mean value of correlation strength is small - close to zero or negative. In the case of particularly long periods of indices grow the correlation strength becomes even smaller than zero, which indicates that during such intervals the returns and normalised daily range time series are converging. What is more, taking into account that the low mean value of correlation strength is accompanied by small standard deviation of correlation strengths it indicates the negative value of correlation strength is observed in the case of most of the analysed time series pairs. At the crisis both mean value and standard deviation of the correlation strength take high values - both parameters are approximately greater than one (few examples of crises are indicated at Figs. 4-7 by red arrows). The high value of standard deviation means that during crises significant differences in correlation strength may be observed. Another important feature of PLCS correlation strength is the fact that the severity of a crisis can be
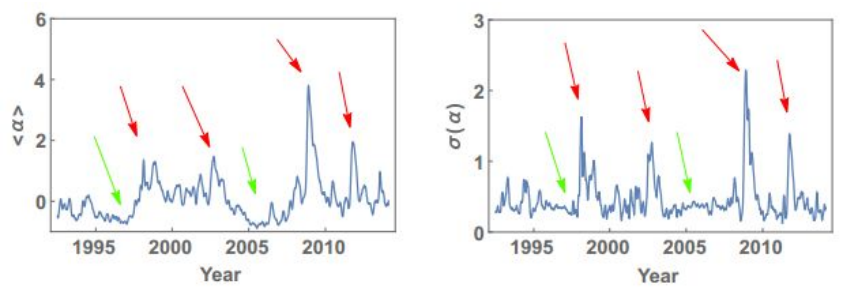

Fig. 4. The evolution of mean value and standard deviation of the correlation strength between daily returns time series of chosen indices. The red arrows indicate examples of crises while the green arrows point some of the prosperity periods. The time window size $T=50 \mathrm{~d}$. Colour online.
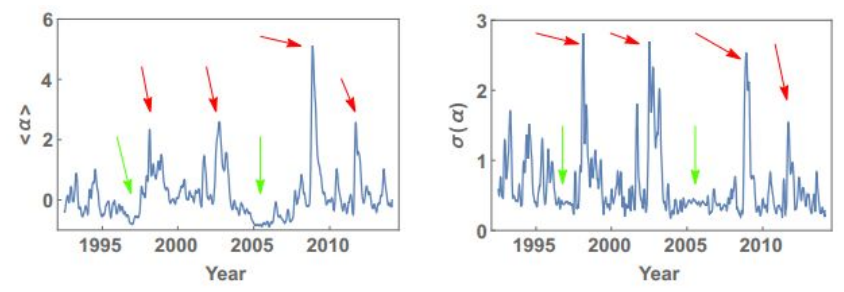

Fig. 5. The evolution of mean value and standard deviation of the correlation strength between normalised daily range time series of chosen indices. The red arrows indicate examples of crises while the green arrows point some of the prosperity periods. The time window size $T=50 \mathrm{~d}$. Colour online.
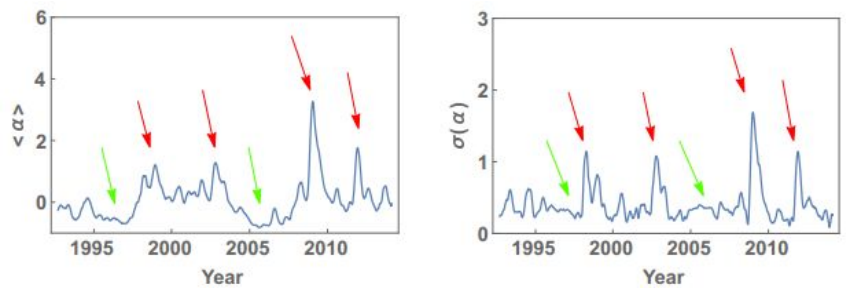

Fig. 6. The evolution of mean value and standard deviation of the correlation strength between daily returns time series of chosen indices. The red arrows indicate examples of crises while the green arrows point some of the prosperity periods. The time window size $T=100 \mathrm{~d}$. Colour online.
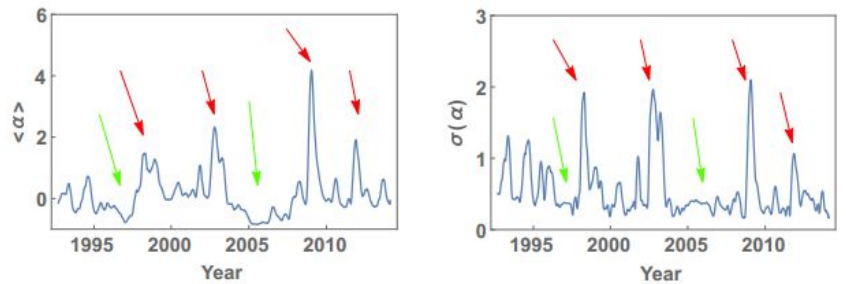

Fig. 7. The evolution of mean value and standard deviation of the correlation strength between normalised daily range time series of chosen indices. The red arrows indicate examples of crises while the green arrows point some of the prosperity periods. The time window size $T=100 \mathrm{~d}$. Colour online. 
classified by the value of the mean correlation strength. The highest peak is observed in 2009, which was the worse recent drop of stock market indices. In the crisis not only the mean correlation strength is high but also the standard deviation takes high values indicating significant differences among correlation strength between investigated time series.

The last aspect analysed in this paper was the influence of the time window size onto the results. Comparing the outcome obtained at $T=50 \mathrm{~d}$ and $T=100 \mathrm{~d}$ (Figs. $4-7$ ) one can observe that extension of the time window size results in smoothing of the mean and standard deviation plots. Additionally the absolute values of the mean and standard deviation are also slightly lowered e.g. the highest peak of the daily returns time series at $T=50 \mathrm{~d}$ $\max (\alpha) \approx 4.8$ while at $T=100 \mathrm{~d} \max (\alpha) \approx 3.4$, similarly for the second type of time series i.e. the normalised daily range time series at $T=50 \mathrm{~d} \max (\alpha) \approx 5.2$ while at $T=100 d \max (\alpha) \approx 4.0$. However, the general conclusion holds for both time window sizes.
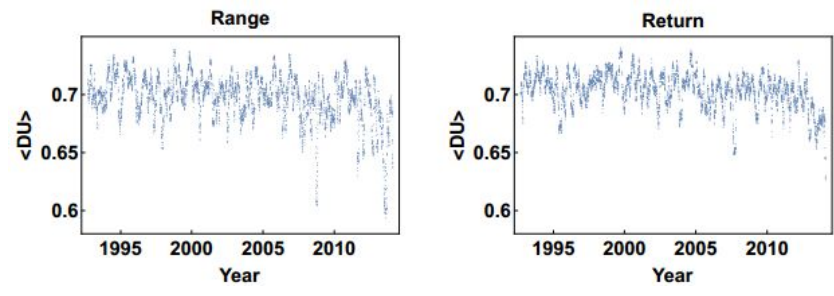

Fig. 8. Evolution of the mean value of UD normalised daily range (left) and daily returns (right) time series in time window size of $50 \mathrm{~d}$.
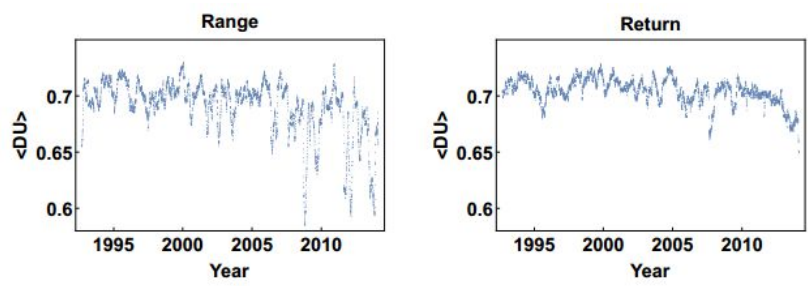

Fig. 9. Evolution of the mean value of UD normalised daily range (left) and daily returns (right) time series in time window size of $100 \mathrm{~d}$.

For the sake of comparison with standard methods the ultrametric distance (Eq. (4)) was used and analogous analysis performed. The evolution of the mean value of UD among daily returns and normalised daily range of stock market indices time series for the time windows sizes $T=50 \mathrm{~d}$ and $T=100 \mathrm{~d}$ were calculated and the results are presented in Figs. 8-9. Although, the standard approach of correlation analysis in crisis is performed on returns time series by the ultrametric distance e.g. $[7,8,10]$ here UD on returns time series averaged over the analysed set of indices and within the chosen time window resulted in outcome where it was difficult to point out the crisis periods. Of course in the detailed analysis one can find out pairs of returns time series where decrease of distances are observed, which denotes increase of correlation. However, after averaging over the whole set these peaks are almost vanishing. Only some of recent serious crises can be recognised (with difficulty) e.g. the significant fall of indices in 2008-2009 resulted in decrease of the mean distance to the value $\langle D U\rangle \approx 0.65$ and $\langle D U\rangle \approx 0.66$ for the time windows $T=50 d$ and $T=100 \mathrm{~d}$ respectively. Surprisingly the UD based analysis of normalised daily range of indices allows to distinguish some of the crisis e.g. that at 2009 for $T=50 \mathrm{~d}$ and 2009, 2011 for $T=100 \mathrm{~d}$. Unfortunately for $T=100 \mathrm{~d}$ mean UD of normalised daily range of indices show one more increase of correlations at 2013, which was rather small drop of stock market indices. Most of the results of the mean value of UD for daily returns indices are in the range $(0.65,0.75)$, that denotes in fact lack of correlations, which is in contrast to observed facts and outcome of PLCS analysis.

\section{Conclusions}

The analysis of cross-correlations among set of chosen stock market indices representing the globally important stock markets was performed by PLCS and their results were compared with analogous UD outcome. The main conclusions are:

- The position of the maxima at the mean value and standard deviation of the correlation strength plots corresponds to the significant drop of the stock markets indices values.

- The low value of mean and standard deviation of the correlation strength corresponds to the prosperity periods at the stock markets.

- The severity of crises are correlated with the height of the mean value peak of the correlation strength in both analysed cases i.e. daily returns and normalised daily range of indices.

- Although further comparative studies are required, the crises were observed if the correlation strength took value above one, while during the prosperity the mean correlation strength was close to zero.

Additional observation made during this study was the fact, that normalized daily range of indices was an promising alternative to the typicaly used daily returns, even in the case of UD. Although the reason might be various, the most probable explanation is that in times of widely available means of communication the most significant changes are observed within the trading day in short range scale rather than on relatively long scale which is a daily returns.

The presented results broaden the possible range of power law classification scheme applications to the analysis of stock markets. However, before it becomes a standard method of the stock market analysis it needs further work in calibration of the method to establish well defined interpretations and advises for traders. 


\section{References}

[1] M. Dash, Tulipomania: The Story of the World's Most Coveted Flower \& the Extraordinary Passions It Aroused, Broadway Books, New York 2001.

[2] J. Miśkiewicz, Physica A 392, 2150 (2013).

[3] J. Miśkiewicz, Acta Phys. Pol. A 123, 589 (2013).

[4] R.N. Mantegna, H.E. Stanley, Introduction to Econophysics: Correlations and Complexity in Finance, Cambridge University Press, 1999.

[5] R.N. Mantegna, Eur. Phys. J. B 11, 193 (1999).
[6] G. Bonanno, F. Lillo, R.N. Mantegna, Physica A 299, 16 (2001).

[7] G. Bonanno, N. Vandewalle, R.N. Mantegna, Phys. Rev. E 62, R7615 (2000).

[8] T. Di Matteo, T. Aste, R.N. Mantegna, Physica A 339, 181 (2004).

[9] J. Miśkiewicz, M. Ausloos, Physica A 387, 6584 (2008).

[10] T. Mizuno, H. Takayasu, M. Takayasu, Physica A 364, 336 (2006). 